\title{
Placental abruption in each phenotype of hypertensive disorders of pregnancy: a retrospective cohort study using a national inpatient database in Japan
}

\author{
Nelson Sass ${ }^{1} \cdot$ Gilberto Nagahama ${ }^{1,2} \cdot$ Henri Augusto Korkes ${ }^{2,3}$ \\ Received: 24 August 2020 / Revised: 2 September 2020 / Accepted: 3 September 2020 / Published online: 7 October 2020 \\ (c) The Japanese Society of Hypertension 2020
}

In a very interesting paper, Naruse et al. [1] analyzed a retrospective cohort from a Japanese database, identifying a higher incidence of placental abruption in women with severe preeclampsia (PE) when admitted at less than 34 weeks gestational age (GA). Abruption of the placenta is an obstetric emergency that is usually catastrophic and requires prompt diagnosis and immediate intervention, usually by emergency cesarean section, to save the fetus and reduce the risk of bleeding complications in the mother. The results from Naruse et al. [1] highlight that this serious condition has occurred after hospital admission in expectant patients with early onset PE at a GA less than 34 weeks.

Worldwide, preeclampsia represents one of the main causes of maternal mortality and a life-threatening situation, especially in low- and middle-income countries. Preeclampsia also results in serious perinatal problems, including fetal death and elective prematurity. At first, considering $\mathrm{PE}$ as a hemorrhagic syndrome does not make sense because PE is classically defined as a hypertensive syndrome, and protocols are focused on controlling blood pressure and planning the best time for delivery. However, severe forms of PE are responsible for hemorrhagic events such as stroke, hepatic hematoma, intensive intravascular coagulation, abruption of the placenta, and abnormal bleeding following delivery.

$\triangle$ Nelson Sass

nelsonsa.alp@terra.com.br

1 Department of Obstetrics, Escola Paulista de Medicina Universidade Federal de São Paulo (UNIFESP), São Paulo (SP), Brazil

2 Hospital Municipal e Maternidade Escola de Vila Nova Cachoeirinha (SMS-SP), São Paulo (SP), Brazil

3 Obstetrics Division, Pontifical Catholic University of Sao Paulo (PUC-SP), São Paulo (SP), Brazil
Therefore, it is plausible to claim that PE is also a "bloody business" (Fig. 1).

Advances in the knowledge of the pathological changes associated with PE, especially in the early onset forms, suggest that poor trophoblastic invasion in the early stages triggers an inflammatory syndrome with abnormal metabolic pathways, including placental release of large amounts of antiangiogenic factors such as soluble forms-like tyrosine kinase-1 (sFlt-1). High plasma levels of these factors hinder the action of vascular endothelial growth factor (VEGF) and placental growth factor (PLGF) on receptors on the endothelium surface, resulting in endothelial dysfunction [2,3].

Endothelial damage results in impaired blood flow autoregulation, microangiopathic hemolysis, and platelet activation. All of these aspects seem to be present in brain dysfunctions in patients with severe PE and eclampsia [4]. These same mechanisms are likely present in multiple organs, such as the kidneys, liver, placenta, and myometrium. In addition to endothelial damage, severe hypertension can lead to microcirculatory disruptions and hemorrhage.

Considering that PE can rapidly develop into severe forms, there is consistent evidence supporting routine recommendations for all women with $\mathrm{PE}$ at $\geq 37$ weeks GA to be managed with an active approach aimed at delivery within $24 \mathrm{~h} \mathrm{[5,6].} \mathrm{For} \mathrm{women} \mathrm{with} \mathrm{severe} \mathrm{forms} \mathrm{of} \mathrm{PE,}$ regardless of gestational age, an active approach is strongly recommended to anticipate delivery as soon as possible. However, many cases of GA at less than 34 GA weeks are managed with an expectant approach due to prematurity. The findings from Naruse and colleagues [1] suggest an additional risk for abruption of the placenta in expectant patients with PE admitted at $<34$ weeks GA (OR, 3.77; 95\% CI, 3.13-4.53) and contribute to clinical practice by adding an important element to be considered in expectant patients.

Obstetric hemorrhage is one of the main causes of maternal mortality worldwide [7]. The main causes of postpartum hemorrhage $(\mathrm{PPH})$ are uterine atony, retained 
Fig. 1 Summary of the pathogenesis of preeclampsia and high risk of hemorrhage

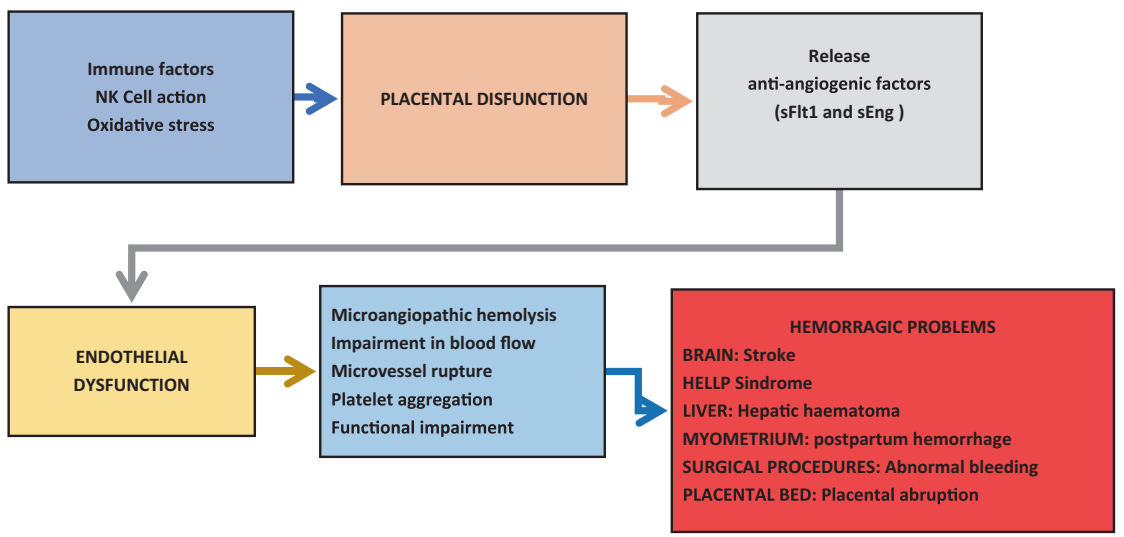

placenta, cesarean delivery, trauma during labor or delivery, abruption of placenta, and coagulation defects. Except for HELLP syndrome, PE is not associated with the risk for $\mathrm{PPH}$. However, the marked endothelial dysfunction associated with $\mathrm{PE}$ can be the initial trigger for a sequence of hemorrhagic events.

A population study conducted in Norway suggested a positive association with $\mathrm{PE}$ and excess bleeding after delivery [8]. According to the authors, the abnormal concentration of antiangiogenic factors (sFlt-1) results in endothelial dysfunction and lack of vasoconstriction after delivery of the placenta. This issue was also the focus of the work of a Nationwide Cohort Study in The Netherlands [9] that identified a prevalence of PPH of $7.4 \%$ in women with PE compared to a prevalence of $4.2 \%$ in women without PE, even after adjusting for confounders. These findings, in addition to the results obtained by Naruse and colleagues [1], increase the awareness of the risks of hemorrhagic events in women with PE in clinical practice.

Endothelial dysfunction could hinder myometrial function, resulting in inadequate contraction following delivery. In addition, many patients can exhibit thrombocytopenia, making hemostasis at the placenta site more difficult. Therefore, care teams need to be aware of the possibility of abnormal bleeding in patients with PE and need to act very quickly. Effective protocols should include planning vaginal delivery, avoiding episiotomy, actively managing the third stage of labor, and making oxytocin and prostaglandin E1 analogs and facilities that allow the immediate administration of fluids and blood products such as whole blood and platelets available. If pharmacological treatment fails, teams must be trained to apply hemostatic sutures through methods such as the B-Lynch technique [10] before deciding to perform a hysterectomy.

We conclude our comments by warning that women with PE may progress to multiple organ failure, including the possibility of severe $\mathrm{PPH}$. These patients are very sensitive to blood loss and need prompt intervention to correct hypovolemia. Thus, PE goes far beyond a hypertensive syndrome, and PE is also a "bloody business".

\section{Compliance with ethical standards}

Conflict of interest The authors declare that they have no conflict of interest.

Publisher's note Springer Nature remains neutral with regard to jurisdictional claims in published maps and institutional affiliations.

\section{References}

1. Naruse K, Shigemi D, Hashiguchi M, Imamura M, Yasunaga H, Arai T. Advanced Life Support in Obstetrics (ALSO)-Japan Research Group. Placental abruption in each hypertensive disorders of pregnancy phenotype: a retrospective cohort study using a national inpatient database in Japan. Hypertens Res. 2020. https:// doi.org/10.1038/s41440-020-00537-6. [Epub ahead of print].

2. Levine RJ, Maynard SE, Qian C, Lim KH, England LJ, Yu KF, et al. Circulating angiogenic factors and the risk of preeclampsia. N. Engl J Med. 2004;350:672-83.

3. Wang A, Rana S, Karumanchi SA. Preeclampsia: the role of angiogenic factors in its pathogenesis. Physiology. 2009;24:147-58.

4. McDermott M, Miller EC, Rundek T, Hurn PD, Bushnell C. Preeclampsia: association with posterior reversible encephalopathy syndrome and stroke. Stroke. 2018;49:524-30.

5. Koopmans CM, Bijlenga D, Groen H, McVijgen S, Aarnoudse $\mathrm{JG}$, Bekedam DJ, et al. Induction of labour versus expectant monitoring for gestational hypertension or mild pre-eclampsia after 36 weeks' gestation (HYPITAT): a multicentre, open-label randomised controlled trial. Lancet. 2009;374:979-88.

6. Chappell LC, Brocklehurst P, Green ME, Hunter R, Hardy P, Juszczak E, et al. Planned early delivery or expectant management for late preterm pre-eclampsia (PHOENIX): a randomised controlled trial. Lancet. 2019;394:1181-90.

7. Osanan GC, Padilla H, Reis MI, Tavares AB. Strategy for zero maternal deaths by hemorrhage in brazil: a multidisciplinary initiative to combat maternal morbimortality. Rev Bras Ginecol Obstet. 2018;40:103-5.

8. Eskild A, Vatten LJ. Abnormal bleeding associated with preeclampsia: a population study of 315,085 pregnancies. Acta Obstet Gynecol Scand. 2009;88:154-8. 
9. von Schmidt auf Altenstadt JF, Hukkelhoven CWPM, van Roosmalen J, Bloemenkamp KWM. Pre-eclampsia increases the risk of postpartum haemorrhage: a nationwide cohort study in The Netherlands. PLoS ONE. 2013;8:e81959.
10. Korkes H, Oliveira LG, Watanabe E, Aoki TT, Ramos CL, Nagahama $\mathrm{G}$, et al. PP022 the haemostatic suture (technique of B-Lynch) may be an alternative to control uterine hemorrhage associated with hypertensive disorders. Pregnancy Hypertens. 2012;2:252-3. 